\title{
Object detection and video coding using background mosaic plane
}

\author{
Ju-Hyun Cho \\ Seong-Dae Kim \\ Korea Advanced Institute of Science \\ and Technology \\ Division of Electrical Engineering \\ Department of Electrical Engineering \\ and Computer Science \\ 373-1, Kusong-dong, Yusong-gu \\ Taejon, 305-701, Republic of Korea \\ E-mail: mainkill@sdvision.kaist.ac.kr
}

\begin{abstract}
Object detection in image sequences has a very important role in many applications, such as surveillance systems, tracking and recognition systems, and coding systems. We are interested in background subtraction, which is very popular algorithm for object detection in image sequences, and also concerned to use the result of detection in selective video coding. Especially when the camera moves and zooms in on something to track the target under drastic illumination change, it is very hard to detect the object properly, and the coding efficiency is reduced. So we generate a multiple background mosaic system, called the background mosaic plane, and use it for object detection and video coding. Some experimental results for both object detection and video coding in various environments show that the average performance of the proposed algorithm is good. () 2007 Society of Photo-Optical Instrumentation Engineers. [DOI: 10.1117/1.2540643]
\end{abstract}

Subject terms: background mosaic plane; resolution change; global illumination change; object detection; video coding.

Paper 050982RR received Dec. 15, 2005; revised manuscript received Aug. 9, 2006; accepted for publication Aug. 15, 2006; published online Feb. 23, 2007.

\section{Introduction}

Object detection in image sequences has a very important role in many research areas related to computer vision. So there have been many researches to solve problems in detecting objects in image sequences. ${ }^{1-18}$ In general object detector should be robust to some noise and has to be adaptive to illumination changes in real environments. It is also very important to detect objects as fast as possible in realtime applications. When the camera moves to track the detected object, global motion estimation (GME) and global motion compensation (GMC) must be done first in general. ${ }^{19-25}$ But with respect to object detection and video coding in image sequences, it is very useful to generate a background mosaic ${ }^{20,24}$ and use it instead of wasting the past parts of the images. In the case of a visual surveillance system, the camera motion is very regular and repeated, so the background mosaic will be more helpful for object detection and video coding. The word "background" means that the mosaic does not include any object. That situation is possible if we use the previous results of object detection. Another reason we use a background mosaic is the following: GME using a background mosaic is more accurate than using the previous image, because there is no induced error from moving objects, and the accumulated GME error during the whole sequence is relatively small.

In generating a background mosaic and using it to detect objects, we observed two serious problems. One is resolution change (RC), and the other is illumination change. These problems are very serious ones in video coding also.

When the camera moves to track the most interesting target, zooming in on the target occasionally occurs in order to get higher resolution. When we generate the new

0091-3286/2007/\$25.00 @ 2007 SPIE background mosaic in that case, loss of information cannot be avoided, because the current image is warped and stitched to the reference background mosaic, which was previously generated and has lower resolution than the current image. In practice the loss of information is very serious with respect to object detection, and it creates large residuals in video coding.

GME between two images has a lot of applications, such as image registration, ${ }^{21-23}$ object detection and tracking, and panorama mosaic generation. Among the related algorithms we focus on optical-flow-based iterative parameter estimation, ${ }^{19,20,24,25}$ which is known to have relatively good performance in some environments. This algorithm assumes that the intensity of a pixel does not change along the motion trajectory-the so-called brightness consistency of the optical flow equation. But this assumption is not true when there are illumination changes between two images, which may be induced by the light source, object motion, camera response, and so on. Especially we are interested in global illumination change (GIC), such as that due to global motion (GM). To handle GIC, we use the popular linear GIC model, ${ }^{25}$

$I_{2}\left(\mathbf{x}^{\prime}\right)=a I_{1}(\mathbf{x})+b$

where $I_{1}(\mathbf{x})$ and $I_{2}\left(\mathbf{x}^{\prime}\right)$ are the intensities at $\mathbf{x}=[x, y]^{T}$ and $\mathbf{x}^{\prime}=\left[x^{\prime}, y^{\prime}\right]^{T}$, respectively, and the GIC parameter $[a b]^{T}$ is constant between the two images.

Some researchers have focused on using illuminationinvariant features, ds in the shading model (SM), ${ }_{17}^{16}$ the method of statistical circular shift moments (SCSM), ${ }^{17}$ and the Wronskian method (WM) ${ }^{18}$ for object detection. However, when the camera moves during illumination change, we should first estimate and compensate the camera motion. But it is very difficult to do that by using the men- 
tioned invariant features. So in this work we use an iterative parameter estimation method, which estimates GM and GIC parameters at the same time to minimize a global cost function, ${ }^{25}$ so it outperforms independent optimization methods. Also, in order to get more accurate parameters in case of outliers (for example, due to moving objects), we use outlier rejection with two stop criteria during the iteration. ${ }^{13}$ But although the GIC is estimated correctly, the compensation error is not negligible for object detection and video coding when there is drastic GIC. We prove that in the next section.

So far, we have mentioned two big problems for object detection and video coding using a background mosaic in image sequences. One is RC and the other is GIC. In this work we generate a multiple background mosaic system, called the background mosaic plane (BMP), and use it for object detection and video coding to overcome these problems. The BMP is made up of many background mosaics, each with a different resolution and illumination level that is dynamically calculated and assigned to it using both a resolution change descriptor (RCD) and a global illumination change descriptor (GICD).

\section{Background Mosaic Plane}

The resolution of images usually changes when the camera moves or zooms in on something to get a higher-resolution image. The acquired high-resolution image can be used in target recognition systems or efficient coding and storage systems. In constructing a mosaic, loss of information cannot be avoided in that case, because the current image is warped and stitched to the reference mosaic, which is previously generated and has lower resolution than the current image. That fact makes it very difficult to detect objects because the background does not have enough information for the purposes of the current image in which coordinate object detection is accomplished. So we need to store and manage information efficiently. For that purpose we propose a BMP, which is made up of many background mosaics, each having a different resolution, to handle drastic RC. In the previous section we explained that GIC as well as GM should be estimated and compensated well for object detection. But although GIC is estimated correctly, the compensation error is not negligible for object detection and video coding when there is drastic GIC, as in Fig. 1. Assume that there is a small compensation error $\delta$ between adjacent images as in

$\left|I_{1}-\left(a_{t-1, t} I_{t-1}+b_{t-1, t}\right)\right|<\delta, \quad t=1,2,3, \ldots$,

where $a_{t-1, t}$ and $b_{t-1, t}$ are GIC parameters between two images, and $I_{t}$ is intensity. If we use $I_{0}$ instead of $I_{1}$ to compensate GIC at $t=2$ as in

$\left|I_{2}-\left(a_{1,2} I_{1}+b_{1,2}\right)\right|<\delta$

$\left|I_{2}-\left(a_{0,2} I_{0}+b_{0,2}\right)\right|<\left(a_{1,2} a_{0,1}+1\right) \delta$,

$a_{0,2}=a_{1,2} a_{0,1}, \quad b_{0,2}=a_{1,2} b_{0,1}+b_{1,2}$,

the compensation error increases because $a_{1,2} a_{0,1}$ is always positive, which means that GIC compensation error gets

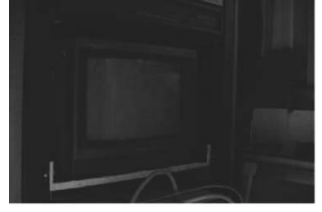

(a)

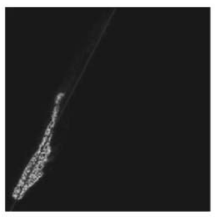

(c)

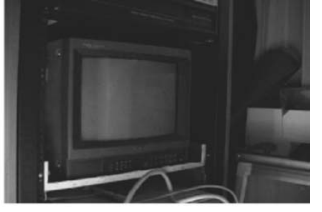

(b)

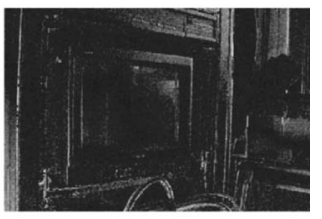

(d)
Fig. 1 GIC compensation error: (a) 1st frame; (b) 100th frame; (c) 2-D cross histogram between (a) and (b); (d) difference picture between (a) and (b) after GIC compensation.

larger as GIC gets stronger. Figure 1 empirically shows that GIC compensation error is very severe in the case of drastic GIC.

So we need a method to reduce the GIC compensation error when there is drastic GIC. Here, the solution is BMP again, because BMP has many background mosaics with each different GIC level to handle drastic GIC as well as drastic RC.

First, we explain the BMP structure in brief, and then we show the workflow of BMP generation. To do that, we must define some descriptors, such as the RC descriptor (RCD) and GIC descriptor (GICD).

\subsection{BMP Structure}

Figure 2 shows the BMP structure. It is made up of many background mosaics which are dynamically generated and aligned along two axes, the representative RC (RRC) axis and the representative GIC (RGIC) axis. Here, "dynamically generated" means that each background mosaic in the BMP is not reused but newly generated if the RRC or RGIC of the current image exceeds the previous maximum or minimum level. The first-generated background mosaic in the BMP is the reference background mosaic positioned at $(\mathrm{RR}, \mathrm{RGIC})=(1,0)$ in Fig. 2. Here RRC and RGIC describe the levels of the current image compared with the reference background mosaic with respect to two important

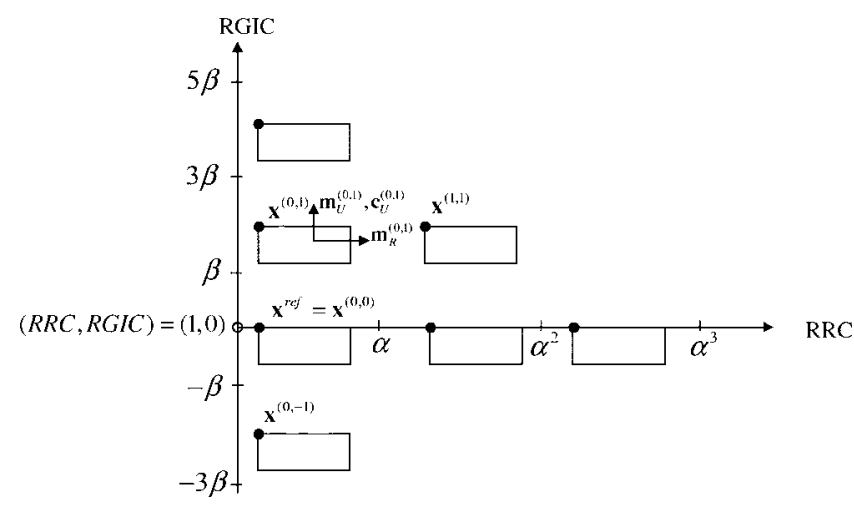

Fig. 2 BMP structure. 
characteristics, RC and GIC. Of course, the two representative changes, RRC and RGIC, are estimated using the corresponding descriptors, RCD and GICD respectively. The descriptors are explained further in the following sections. Here $\mathbf{x}^{(i, j)}$ is the background mosaic coordinate positioned at $(i, j)$ in the BMP, and $\mathbf{m}_{R}^{(i, j)}, \mathbf{m}_{U}^{(i, j)}$, and $\mathbf{c}_{U}^{(i, j)}$ are $\mathrm{GM}$ and GIC parameters positioned at $(i, j)$ and referring to the right and upward directions, respectively, which are estimated between the adjacent background mosaics in each direction. In particular, the reference background mosaic with the coordinate $\mathbf{x}^{\text {ref }}=\mathbf{x}^{(0,0)}$ lies at $(\mathrm{RR}, \mathrm{RGIC})=(1,0)$, which is the reference point of the BMP. The quantities $\alpha^{m}$ and $\beta n$ are the quantized RC Level (RCL) and GIC level (GICL), respectively, at which one background mosaic can overcome the given RC and GIC. Then a new background mosaic in the BMP is generated dynamically if necessary, as mentioned before, i.e., the BMP needs a new background mosaic to handle the given RC and GIC. So a BMP is generated during object detection with the use of background mosaics. We should first find the corresponding background mosaic to the BMP by estimating the RRC and RGIC of the current image. That mosaic has the most similar RRC and RGIC to those of the current image, so is suitable for object detection. The method to choose it is shown in detail below.

\subsection{Resolution Change Descriptor}

The RCD is a descriptor to express the RC between the reference background mosaic and the current image, which is defined and calculated at reference background mosaic coordinate by ${ }^{24}$

$\mathbf{x}=\mathbf{W}\left(\mathbf{x}^{\mathrm{ref}} ; \mathbf{m}\right)$

$\operatorname{RCD}\left(\mathbf{x}^{\mathrm{ref}}\right)=\left[\begin{array}{ll}\left|\partial x / \partial x^{\mathrm{ref}}\right| & \left|\partial x / \partial y^{\mathrm{ref}}\right| \\ \left|\partial y / \partial x^{\mathrm{ref}}\right| & \left|\partial y / \partial y^{\mathrm{ref}}\right|\end{array}\right]$,

where $\mathbf{W}\left(x^{\text {ref }} ; \mathbf{m}\right)$ is a warping function between the reference background mosaic coordinate $\mathbf{x}^{\text {ref }}$ and the current image coordinate $\mathbf{x}$. It is defined at each pixel and describes the instantaneous resolution change. We want to get a RRC of the current image compared to the reference background mosaic, which we do as follows.

If we use a planar model

$$
\begin{aligned}
& \mathbf{x}=\left[\begin{array}{ll}
x & y
\end{array}\right]^{T}, \quad \mathbf{x}^{\prime}=\left[\begin{array}{ll}
x^{\prime} & y^{\prime}
\end{array}\right]^{T}, \\
& \mathbf{m}=\left[\begin{array}{llll}
m_{0} & m_{1} & \ldots & m_{7}
\end{array}\right]^{T}, \\
& x^{\prime}=\frac{\left(1+m_{0}\right) x+m_{1} y+m_{2}}{m_{6} x+m_{7} y+1}, \quad y^{\prime}=\frac{m_{3} x+\left(1+m_{4}\right) y+m_{5}}{m_{6} x+m_{7} y+1},
\end{aligned}
$$

then the RCD becomes

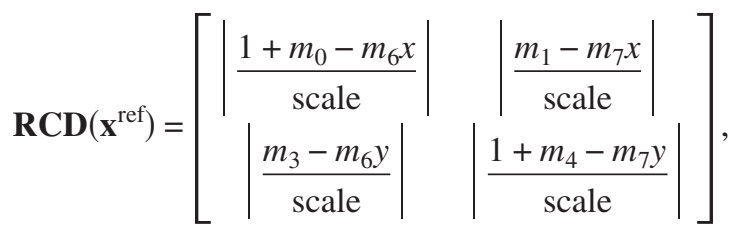

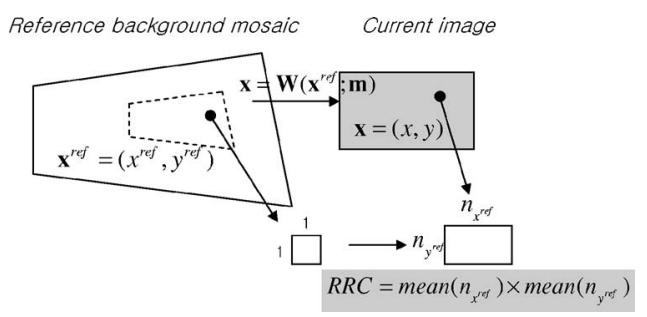

Fig. 3 Representative resolution change (RRC) calculation using resolution change descriptor $(R C D)$ at each pixel of the reference background mosaic.

scale $=m_{6} x^{\text {ref }}+m_{7} y^{\text {ref }}+1$.

This planar model uses eight parameters and normalizes scalability with a perspective transformation, which is called the homography.

Now we can get the needed number of pixels in the current image to express that amount of $\mathrm{RC}$, for which we propose the following RRC:

$$
\begin{aligned}
& \mathbf{n}\left(\mathbf{x}^{\text {ref }}\right)=\left[\begin{array}{ll}
n_{x^{\mathrm{ref}}} & n_{y^{\mathrm{ref}}}
\end{array}\right]^{T}=\mathbf{R C D}\left(\mathbf{x}^{\mathrm{ref}}\right) \mathrm{d} \mathbf{x}^{\mathrm{ref}}, \\
& \mathbf{d} \mathbf{x}^{\mathrm{ref}}=\left[\begin{array}{ll}
1 & 1
\end{array}\right]^{T}, \\
& \operatorname{mean}\left(\mathbf{n}\left(\mathbf{x}^{\mathrm{ref}}\right)\right)=\left[\operatorname{mean}\left(n_{x^{\mathrm{ref}}}\right) \quad \operatorname{mean}\left(n_{y^{\mathrm{ref}}}\right)\right]^{T}, \\
& \mathrm{RRC}=\operatorname{mean}\left(n_{x^{\mathrm{ref}}}\right) \times \operatorname{mean}\left(n_{y^{\mathrm{ref}}}\right),
\end{aligned}
$$

as shown in Fig. 3, where $n_{x^{\mathrm{ref}}} \times n_{y^{\mathrm{ref}}}$ is the number of pixels needed to express that amount of $\mathrm{RC}$ at $\mathbf{x}$ in the current image if the corresponding number of pixels is $1 \times 1$ at $\mathbf{x}^{\mathrm{ref}}$. Here, we want to find the RRC, a representative $\mathrm{RC}$ in the current image, so we calculate the mean of $\mathbf{n}\left(\mathbf{x}^{\mathrm{ref}}\right)$ and use it to get the RRC. In other words, the RRC is the average number of pixels needed to express the resolution change between the reference background mosaic and the current image. Figure 4(b) shows the RRC and quantized RC level (RCL) through an image sequence; the RCL is described in detail below. We find that the resolution of the last image is about 8 times as high as the first one, i.e., the camera has zoomed in a lot, as seen in Fig. 4(a).

\subsection{Global Illumination Change Descriptor}

The GICD is a descriptor to express the GIC between the reference background mosaic and the current image, which is defined and calculated at reference background mosaic coordinate as follows:

$\mathbf{x}=\mathbf{W}\left(\mathbf{x}^{\mathrm{ref}} ; \mathbf{m}\right)$

$I(\mathbf{x})=G\left(I\left(\mathbf{x}^{\mathrm{ref}}\right) ; \mathbf{c}\right)$

$\operatorname{GICD}\left(I\left(\mathbf{x}^{\mathrm{ref}}\right)\right)=I(\mathbf{x})-I\left(\mathbf{x}^{\mathrm{ref}}\right)$,

where $\mathbf{W}\left(\mathbf{x}^{\text {ref }} ; \mathbf{m}\right)$ is a warping function and $G\left(I\left(\mathbf{x}^{\text {ref }}\right) ; \mathbf{c}\right)$ is a GIC function between the reference background mosaic 

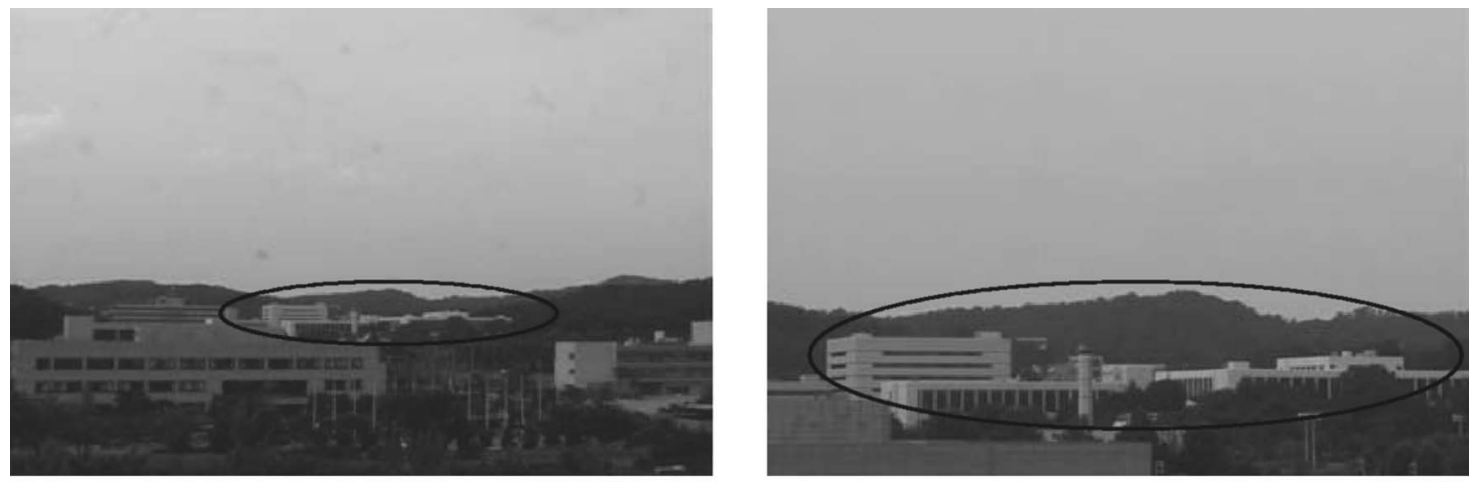

(a)

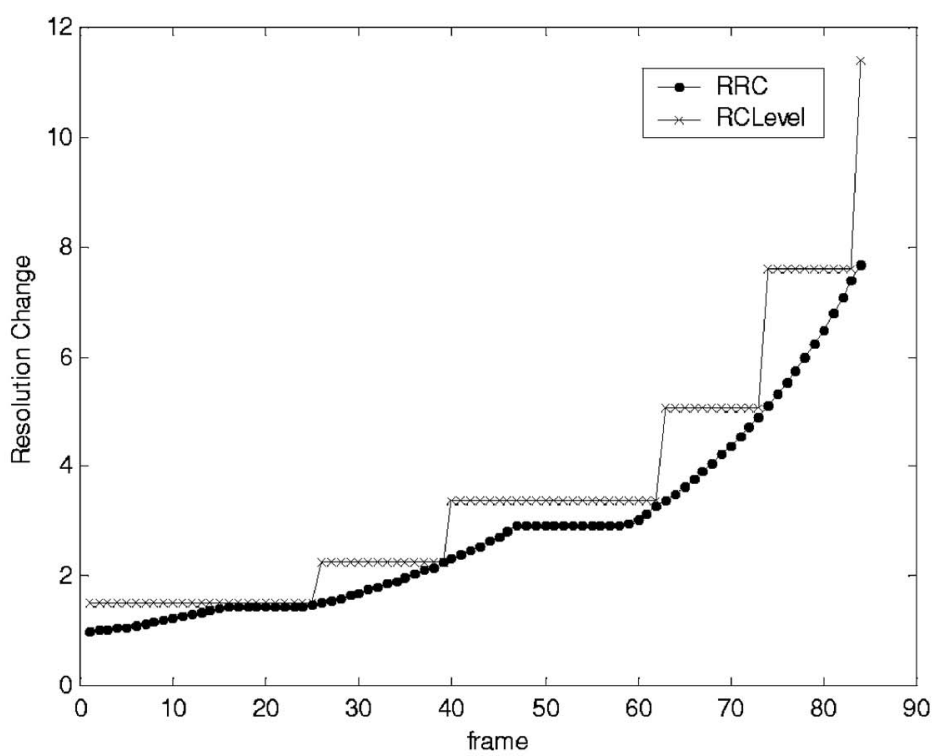

(b)

Fig. 4 The resolution change in an image sequence: (a) images with drastic resolution change, (b) the resolution change. In (a) the left image is the 1st frame and the right one is the 85th frame in the sequence. In (b) the dotted line represents the RRC, and crossed line shows the corresponding RCL.

coordinate $\mathbf{x}^{\text {ref }}$ and the current image coordinate $\mathbf{x}$ as given in Eq. (5) and in

$$
G\left(I_{1}(\mathbf{x})\right)=\left(1+c_{0}\right) I_{1}(\mathbf{x})+c_{1},
$$$$
\mathbf{c}=\left[\begin{array}{ll}
c_{0} & c_{1}
\end{array}\right]^{T},
$$

respectively. The GICD is defined at each pixel and describes instantaneous illumination change. Here, we want to find RGIC, a representative GICD in the current image like the RRC, so we calculate the mean of $\operatorname{GICD}\left(I\left(\mathbf{x}^{\mathrm{ref}}\right)\right)$ and use it to get

$\operatorname{RGIC}=\operatorname{mean}\left(\operatorname{GICD}\left(I\left(\mathbf{x}^{\mathrm{ref}}\right)\right)\right)=\frac{1}{256} \sum_{I\left(\mathbf{x}^{\mathrm{ref}}\right)=0}^{255}\left[I(\mathbf{x})-I\left(\mathbf{x}^{\mathrm{ref}}\right)\right]$,

as shown in Fig. 5. In other words, the RGIC is the averaged illumination change between the reference background mosaic and the current image. Figure 6(b) shows

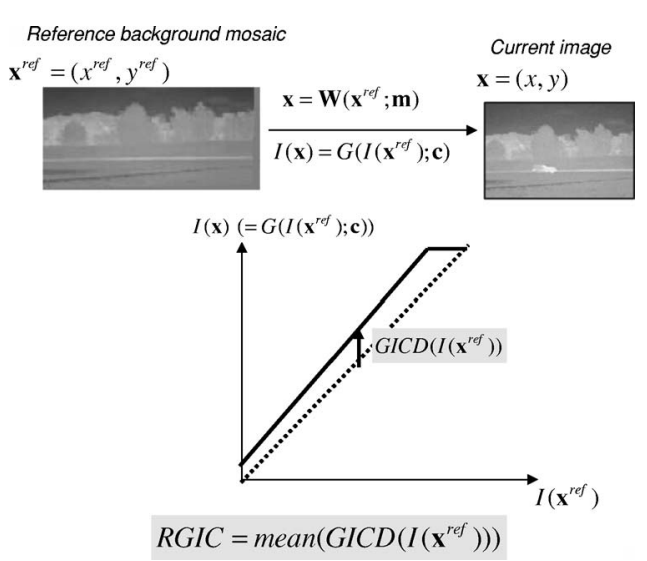

Fig. 5 Representative global illumination change (RGIC) calculation using global illumination change descriptor (GICD) at each pixel of the reference background mosaic. 

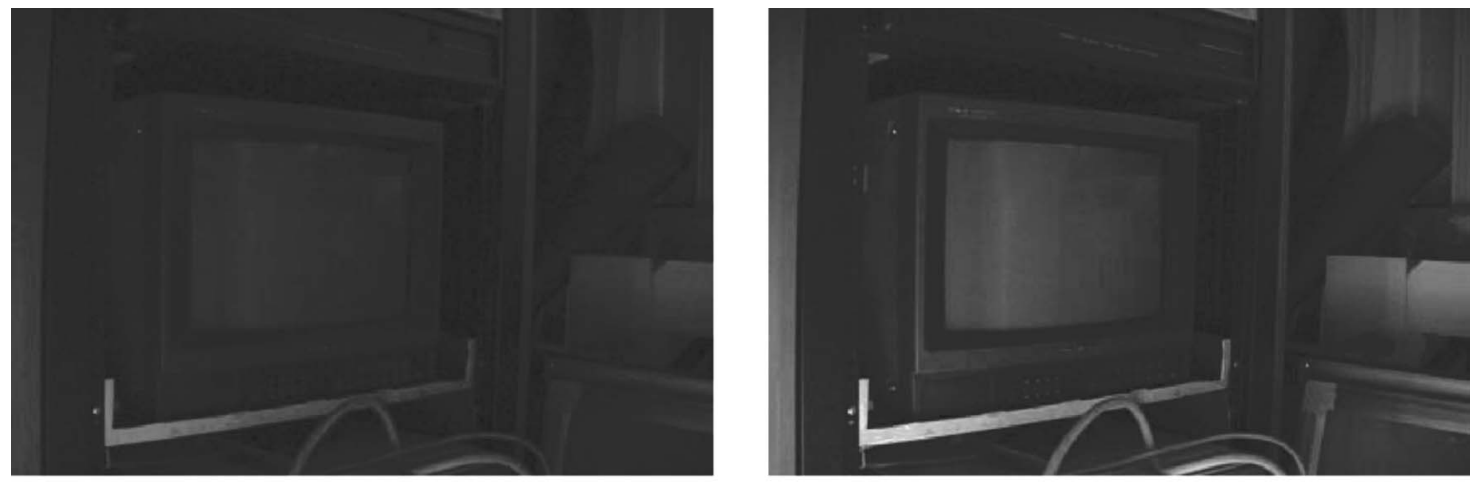

(a)

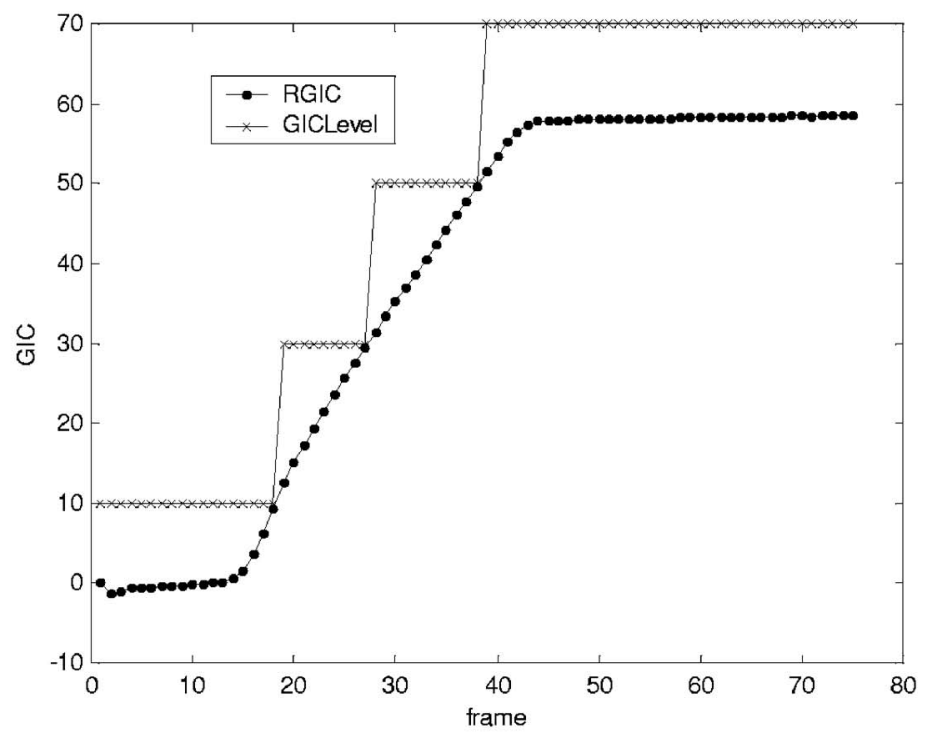

(b)

Fig. 6 The illumination change in an image sequence: (a) images with drastic illumination change, (b) the illumination change. In (a) the left image is the 1st frame and the right one is the 75th frame in the sequence. In (b) the dotted line represents the RGIC, and the crossed line shows the corresponding GICL.

the RGIC and quantized GIC level (GICL) through an image sequence; the GICL is described in detail below. We find that the last image is much brighter than the first one because a light was turned on, as seen in Fig. 6(a).

\subsection{BMP Generation}

Considering the results of some experiments on object detection and video coding, we first define quantized RCL and GICL as

$\mathrm{RCI}_{m}=\alpha^{m} \quad(m=1,2,3, \ldots)$,

$\mathrm{GICL}_{n}=(2 n+1) \beta \quad(n=0, \pm 1, \pm 2, \pm 3, \ldots)$,

where $\alpha$ and $\beta$ are constants to be determined, and we assign these levels to each background mosaic in the BMP as shown in Fig. 2. Here, RCL and GICL show the capability of each background mosaic to handle the amounts of changes, RC and GIC, respectively. For example, if a background mosaic has the levels $\mathrm{RCL}_{2}$ and $\mathrm{GICL}_{1}$, then the mosaic can handle the corresponding amounts of RC and GIC, i.e., $\alpha \leq \mathrm{RC}<\alpha^{2}$ and $\beta \leq \mathrm{GIC}<3 \beta$. This means that the RRC of the current image in that RCL can be from $\alpha$ to $\alpha^{2}$ times the resolution of the reference background mosaic. Also, the RGIC of the current image in that GICL can be larger than the illumination level of the background mosaic by a factor from $\beta$ to $3 \beta$.

If $\alpha$ is too large, there is a serious problem for object detection, because too much loss of information may occur on that RCL. On the other hand, if $\alpha$ is too small, we need a lot of memory to store and maintain the background mosaics in BMP. From the simulation results, we chose $\alpha$ to be 1.5 typically. The parameter $\beta$ determines the GIC compensation error that the BMP can overcome. If $\beta$ is too large, the compensation error increases, and the resulting object detection is not good. Too small $\beta$ will require a lot of memory on the other hand. We chose $\beta=10$ on the basis of experiments like those in the case of $\alpha$. All the experimental results in this paper were obtained using $\alpha=1.5$ and 


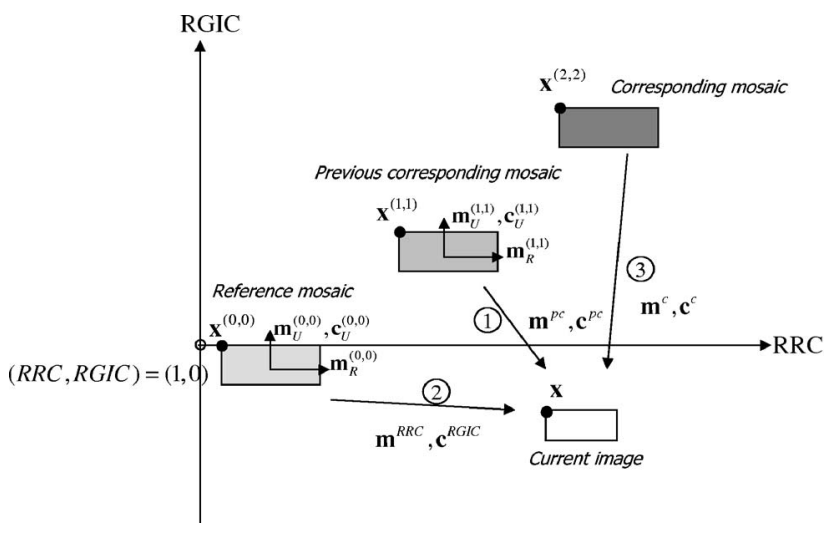

Fig. 7 BMP generation. The circled numbers show the order of processing. $\beta=10$. Of course, a theoretically proved method to choose the optimal $\alpha$ and $\beta$ would be better than ad hoc parameter tuning, which is open to challenge.

As mentioned before, we assume that the BMP generation starts from the reference background mosaic with levels $\mathrm{RCL}_{1}$ and $\mathrm{GICL}_{0}$. If the RRC of the current image exceeds $\alpha$, then a new background mosaic positioned at $(1,0)$ in the BMP will be generated, which is initialized by the values from the previous corresponding background mosaic. In this case, the previous corresponding background mosaic, which was involved in object detection just before, is the reference background mosaic. On the other hand, if there is only the reference background mosaic in $\mathrm{BMP}$ and if the RGIC of the current image exceeds $\beta$ or is lower than $-\beta$, then a new background mosaic positioned at $(0,1)$ or $(0,-1)$ in the BMP will be generated. In that case, the previous corresponding background mosaic is also the reference one. Assume that a more general case occurs. The previous corresponding background mosaic is positioned at
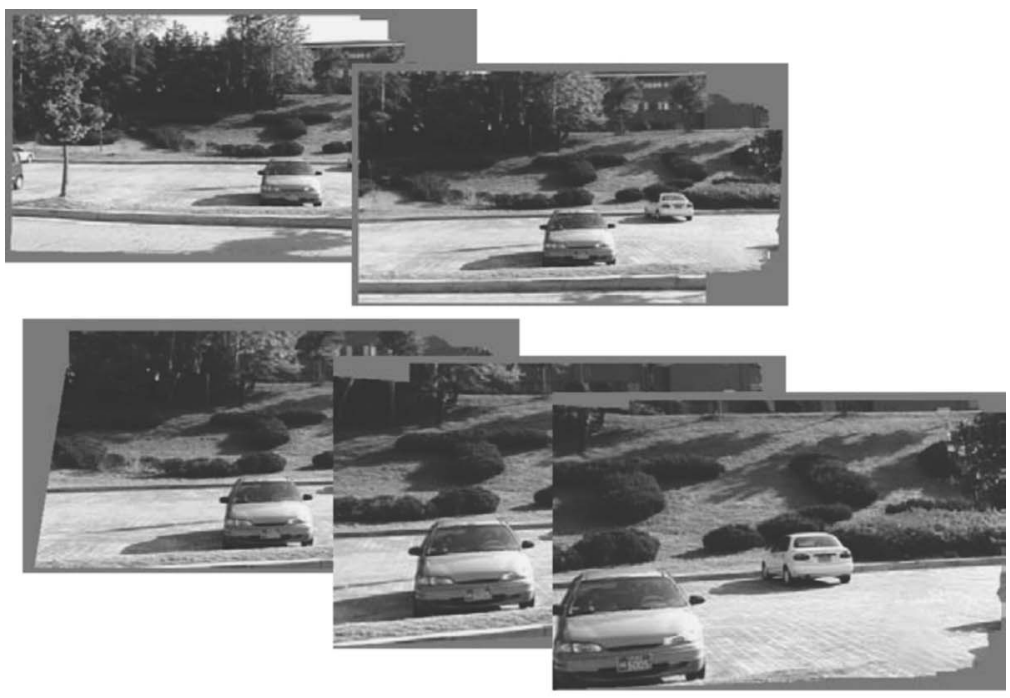

(a)

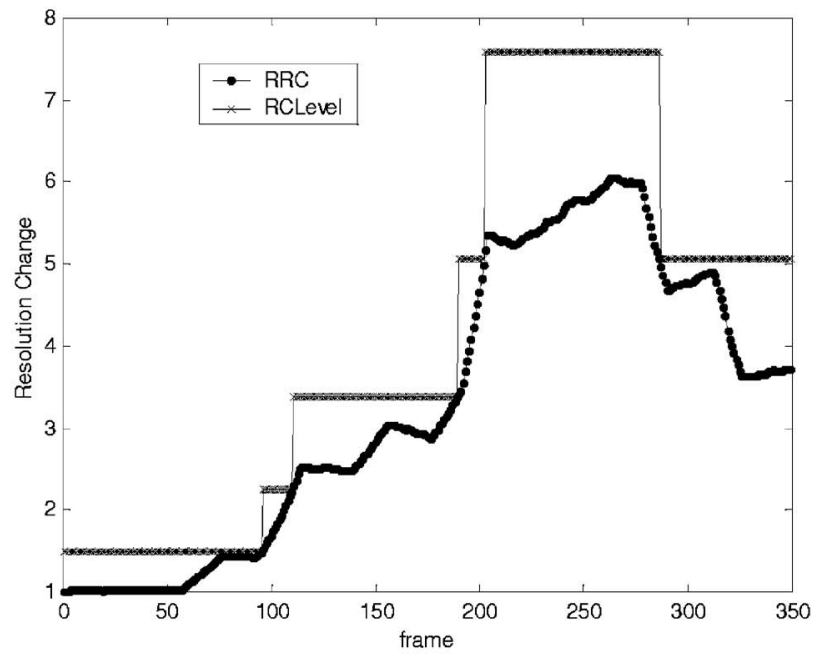

(b)

Fig. 8 BMP with five resolution change levels in the case of fixed global illumination change level: (a) generated background mosaics in BMP, (b) the change of resolution in an image sequence. 


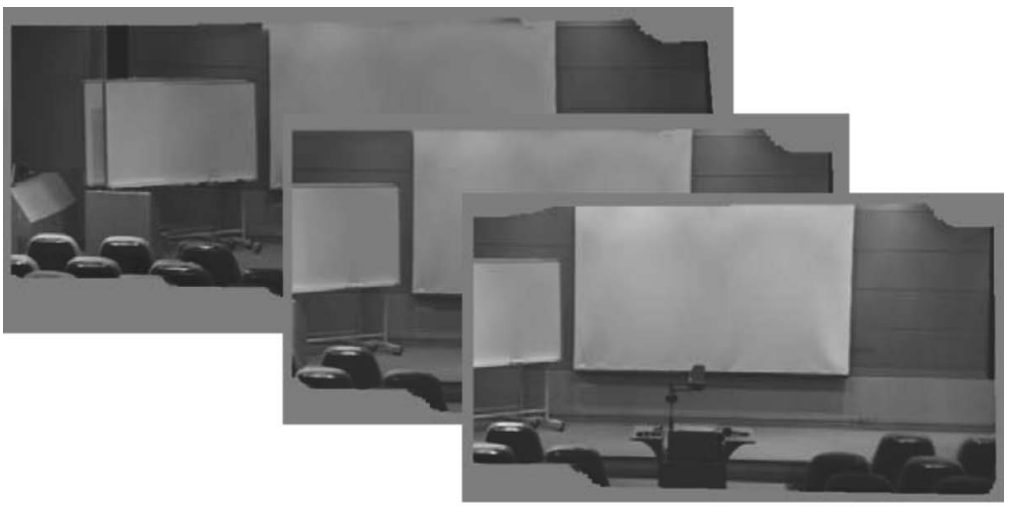

(a)

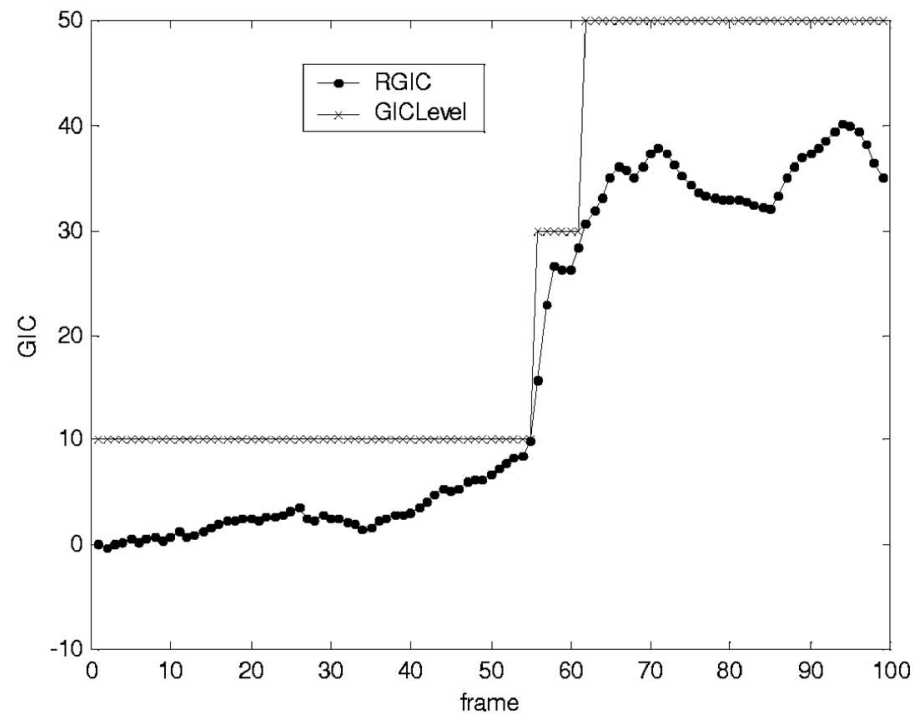

(b)

Fig. 9 BMP with three global illumination change levels in the case of fixed resolution change level: (a) generated background mosaics in BMP, (b) change of illumination in an image sequence.

$(1,1)$, and the calculated corresponding background mosaic is at $(2,2)$, as in Fig. 7. To generate the BMP we should first estimate GM and GIC between the previous corresponding background mosaic and the current image. Then the RRC and RGIC are calculated, and finally the corresponding background mosaic is determined as in Fig. 7, using Eqs. (7) and (10) and

$\mathbf{x}=\mathbf{W}\left(\mathbf{x}^{(i, j)} ; \mathbf{m}\right)=\mathbf{M} \mathbf{x}^{(i, j)}$,

$I(\mathbf{x})=G\left(I\left(\mathbf{x}^{(i, j)}\right) ; \mathbf{c}\right)=\mathbf{C} I\left(\mathbf{x}^{(i, j)}\right) ;$

if $\mathrm{pc}=(1,1)$ and $\mathrm{c}=(2,2)$,

$\mathbf{m}^{\mathrm{pc}} \Rightarrow \mathbf{M}^{\mathrm{RRC}}=\mathbf{M}^{\mathrm{pc}} \mathbf{M}_{U}^{(1,0)} \mathbf{M}_{R}^{(0,0)} \Rightarrow \mathbf{m}^{\mathrm{RRC}}, \quad \mathrm{RRC}=2$,

$\mathbf{c}^{\mathrm{pc}} \Rightarrow \mathbf{C}^{\mathrm{RGIC}}=\mathbf{C}^{\mathrm{pc}} \mathbf{C}_{U}^{(0,0)} \Rightarrow \mathbf{c}^{\mathrm{RGIC}}, \quad \mathrm{RGIC}=2$,

$$
\begin{aligned}
& \mathbf{M}^{\mathrm{c}}=\mathbf{M}^{\mathrm{pc}}\left(M_{R}^{(1,1)}\right)^{-1}\left(\mathbf{M}_{U}^{(2,1)}\right)^{-1} \Rightarrow \mathbf{m}^{c}, \\
& \mathbf{C}^{\mathrm{c}}=\mathbf{C}^{\mathrm{pc}}\left(\mathbf{C}_{U}^{(1,1)}\right)^{-1} \Rightarrow \mathbf{c}^{c}
\end{aligned}
$$

in the case $\mathrm{pc}=(1,1)$ and $\mathrm{c}=(2,2)$, where $\mathbf{m}^{\mathrm{pc}}$ and $\mathbf{c}^{\mathrm{pc}}$ are the previous corresponding GM and GIC parameters, and $\mathbf{m}^{\text {RRC }}$ and $\mathbf{c}^{\text {RGIC }}$ are the parameters that are used in Eqs. (7) and (10) to determine RRC and RGIC, respectively. The calculated $\mathbf{m}^{\mathrm{c}}$ and $\mathbf{c}^{\mathrm{c}}$ are the corresponding GM and GIC parameters between the corresponding background mosaic and the current image. Those are directly used for object detection and video coding. Here, $\mathbf{M}$ is a matrix adopted in order to describe the relation between each coordinate and the GM parameter $\mathbf{m}$ more easily, and $\mathbf{C}$ is a matrix to represent the GIC function in terms of the GIC parameter $\mathbf{c}$. The various subscripts for those are exactly matched with the symbols in Fig. 7. For example, $\mathbf{M}_{R}^{(1,1)}$ represents $\mathbf{m}_{R}^{(1,1)}$, which is the right-direction GM parameter positioned at $(1,1)$ in the BMP. After object detection, we should update the BMP. All the background mosaics with lower RCL than 


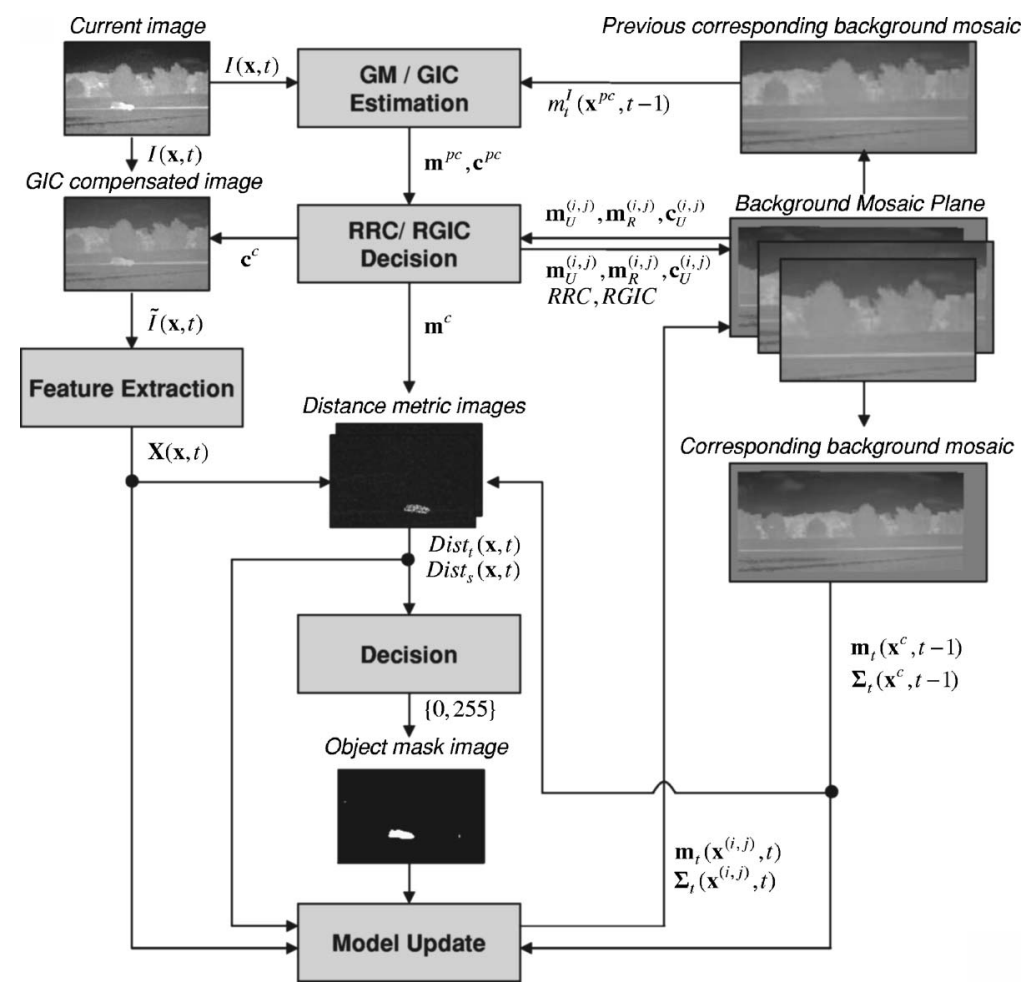

Fig. 10 Overall object detection system.

the RRC of the current image in the BMP are updated after GM and GIC compensation, using the current image and all the recalculated parameters between each background mosaic and the current image. If the resolution of the current image is lower than that of a background mosaic in the BMP, then we can say that the mosaic has better information than the current image. So we had better not use the information of the current image in updating the BMP.

\subsection{Experimental Results}

Figure 8 shows generated background mosaics in the BMP with $\alpha=1.5$ and fixed GICL. The image sequence was obtained using a moved handheld camera, which was zooming in and out throughout the sequence. The BMP has five background mosaics with five different RCLs. Figure 8(b) shows the change of resolution in the sequence. In this case we failed to generate a proper single background mosaic, because the resolution gap was too large between the background mosaic and the current image with the highest resolution, i.e., we could not even estimate the correct GM parameter.

Figure 9 shows generated background mosaics in the BMP with $\beta=10$ and fixed RCL. The sequence was obtained using handheld camera when there was drastic illumination change, viz., a light was turned on. The BMP has three background mosaics with three different GICLs. Figure 9(b) shows the change of global illumination in the sequence. In that case, we could observe that GIC compensation error increased when we used a single background mosaic even if the GIC parameter was estimated properly.

\section{Object Detection using the BMP}

Object detection algorithms in image sequences usually use the temporal information between the images. Among them, methods based on the difference picture (DP) ${ }^{1-14}$ have been extensively developed because they satisfy almost all requirements for object detector, viz., robustness to noise, adaptability to illumination changes, and fast detection. Background subtraction, ${ }^{1-9}$ which uses DP between the current and the background image, is the most popular such method. We proposed a unified framework for background subtraction, ${ }^{13}$ which is made up of three criteria. Two of them are about the feature and distance metrics, respectively, and the third is about the rule for adaptation of the background model to illumination changes. The proposed algorithm ${ }^{13}$ uses spatiotemporal thresholding for object detection with a spatiotemporal distance metric. The distance metric is generated by using the intensity and gradient at the same time on the feature level instead of on the decision level. In model update process we use the truncated variable adaptation rate (TVAR), which can control the adaptation rate (AR) according to its statistics, so it is able to maintain the model's statistics properly through the whole sequence.

\subsection{Overall System}

Here, we are going to describe the workflow of the proposed object detection system in brief. The overall system and and brief description of used symbols are shown in Fig. 10 and Table 1, respectively. The BMP is composed of many background mosaics with different resolutions and illumination levels. The system starts from one background 
(a)

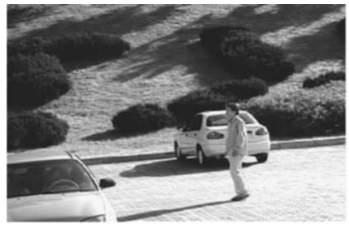

(b)

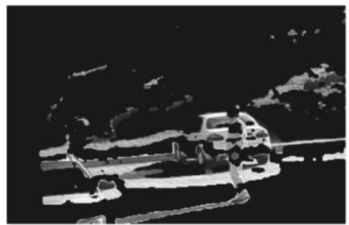

(c)
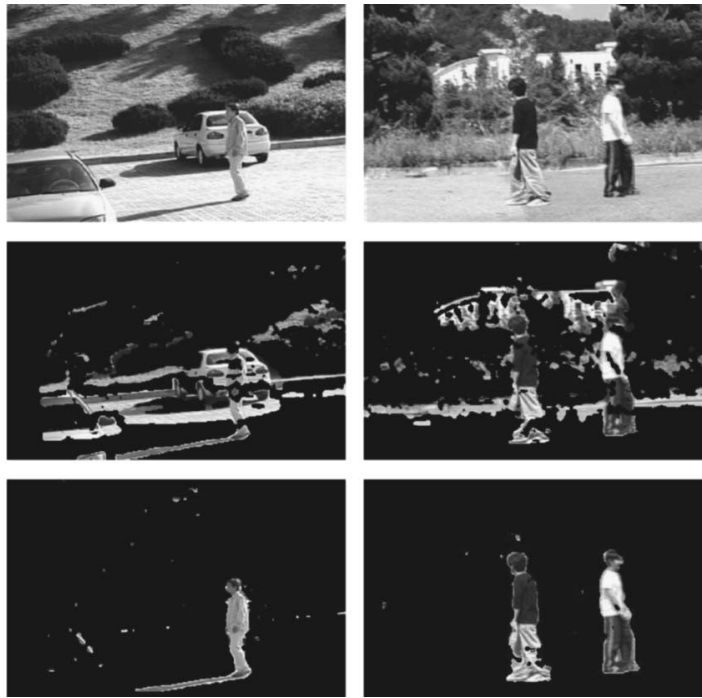

Fig. 11 Object detection using BMP in the case of drastic resolution change: (a) current image, (b) detection result using a single background mosaic, (c) detection result using BMP.

mosaic, which is called the reference background mosaic, and generates another one using a rule as mentioned in Sec. 2. So the number of background mosaics is not to be determined in advance, but increases according to the resolution or illumination changes of the current image. Each background mosaic in the BMP has mean and covariance images having the same dimension as the feature used. After initialization, those mean and covariance images are used to detect objects and are updated according to some criteria.

The proposed system is a recursive one, so the previous result is the starting point of a new iteration. We first estimate GM and GIC parameters between the previous corresponding mosaic and the current image. Here we need to explain again the meaning of corresponding mosaic. "Corresponding" means that it has the most similar resolution and illumination level to that of the current image among all the mosaics in the BMP. So the corresponding mosaic is suitable for object detection, and must be selected from the BMP with the chosen RRC and RGIC. Here, RRC and RGIC were fully explained in the previous section. With the recalculated corresponding GM parameter $\mathbf{m}^{\mathrm{c}}$ and extracted feature $\mathbf{X}(\mathbf{x}, t)$, we generate spatiotemporal distance metric $\operatorname{Dist}_{t}(\mathbf{x}, t)$ and $\operatorname{Dist}_{s}(\mathbf{x}, t) .{ }^{13}$ Before extracting the fea- $^{2}$ ture, we compensate for GIC in the current image with the estimated GIC parameter $\mathbf{c}^{\mathrm{c}}$. Of course, the mean and covariance images of the corresponding background mosaic, $\mathbf{m}_{t}\left(\mathbf{x}^{\mathrm{c}}, t-1\right)$ and $\boldsymbol{\Sigma}_{t}\left(\mathbf{x}^{\mathrm{c}}, t-1\right)$, are also used to generate the distance metric. After thresholding with the spatiotemporal distance metric, an object mask image is generated. Finally, the BMP is updated with some criteria using detection results.

\subsection{Experimental Results}

Figure 11 shows the effect of the BMP in the case of drastic resolution change for object detection. Two sequences were obtained from a handheld CCD camera, and there is zooming in and out as well as camera motion in both sequences. For the first sequence, object detection is accomplished us-
Table 1 Description of symbols in Fig. 10, which shows the proposed overall object detection system.

\begin{tabular}{|c|c|}
\hline Symbol & Description \\
\hline $\mathbf{x}$ & Current image coordinate \\
\hline $\mathbf{x}^{\mathrm{pc}}$ & $\begin{array}{l}\text { Previous corresponding background } \\
\text { mosaic coordinate }\end{array}$ \\
\hline $\mathbf{x}^{\mathrm{c}}$ & $\begin{array}{c}\text { Corresponding background mosaic } \\
\text { coordinate }\end{array}$ \\
\hline $\mathbf{x}^{(i, j)}$ & $\begin{array}{l}\text { Each background mosaic coordinate } \\
\text { with different resolution and } \\
\text { illumination level in BMP }\end{array}$ \\
\hline$I(\mathbf{x}, t)$ & Intensity at $t$ \\
\hline $\bar{I}(\mathbf{x}, t)$ & GIC-compensated intensity at $t$ \\
\hline $\mathbf{X}(\mathbf{x}, t)$ & $\begin{array}{c}\text { Feature using intensity and gradient at } \\
\text { the same time at } t\end{array}$ \\
\hline $\operatorname{Dist}_{t}(\mathbf{x}, t)$ & Temporal distance metric at $t$ \\
\hline $\operatorname{Dist}_{s}(\mathbf{x}, t)$ & Spatial distance metric at $t$ \\
\hline$m_{t}^{\prime}\left(\mathbf{x}^{\mathrm{pc}}, t-1\right)$ & $\begin{array}{l}\text { Intensity component in the temporal } \\
\text { mean of previous corresponding } \\
\text { background mosaic at } t-1\end{array}$ \\
\hline $\mathbf{m}_{t}\left(\mathbf{x}^{\mathrm{c}}, t-1\right)$ & $\begin{array}{l}\text { Temporal mean of corresponding } \\
\text { background mosaic at } t-1\end{array}$ \\
\hline$\Sigma_{t}\left(\mathbf{x}^{\mathrm{c}}, t-1\right)$ & $\begin{array}{c}\text { Temporal covariance of corresponding } \\
\text { background mosaic at } t-1\end{array}$ \\
\hline $\mathbf{m}_{t}\left(\mathbf{x}^{(i, j)}, t\right)$ & $\begin{array}{l}\text { Temporal mean of each background } \\
\text { mosaic with different resolution and } \\
\text { illumination level in BMP at } t\end{array}$ \\
\hline $\mathbf{\Sigma}_{t}\left(\mathbf{x}^{(i, j)}, t\right)$ & $\begin{array}{c}\text { Temporal covariance of each } \\
\text { background mosaic with different } \\
\text { resolution and illumination level in } \\
\text { BMP at } t\end{array}$ \\
\hline $\mathbf{m}^{\mathrm{pc}}, \mathbf{c}^{\mathrm{pc}}$ & $\begin{array}{l}\text { GM and GIC parameters between the } \\
\text { corresponding background mosaic and } \\
\text { the current image }\end{array}$ \\
\hline $\mathbf{m}^{\mathrm{c}}, \mathbf{c}^{\mathrm{c}}$ & $\begin{array}{l}\text { GM and GIC parameters between the } \\
\text { previous corresponding background } \\
\text { mosaic and the current image }\end{array}$ \\
\hline $\mathbf{m}_{R}^{(i, j)}, \mathbf{m}_{U}^{(i, j)}, \mathbf{c}_{U}^{(i, j)}$ & $\begin{array}{l}\text { GM and GIC parameters between } \\
\text { background mosaics in BMP }\end{array}$ \\
\hline $\mathrm{RRC}$ & $\begin{array}{l}\text { Representative resolution change of } \\
\text { the current image }\end{array}$ \\
\hline RGIC & $\begin{array}{l}\text { Representative global illumination } \\
\text { change of the current image }\end{array}$ \\
\hline
\end{tabular}

ing each BMP, which is shown as Fig. 8(a). In this case, we have five RC levels, and the estimated resolution change is shown in Fig. 8(b). The situation for the second sequence is almost same as the first one. The result using a single background model as in Fig. 11(b) reveals that it cannot over- 
(a)

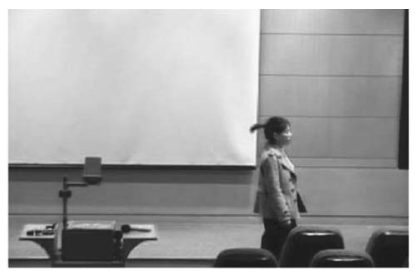

(b)
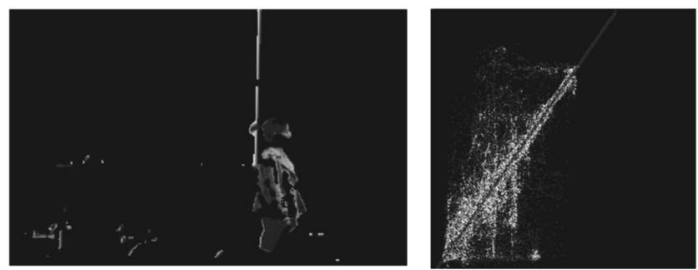

(c)
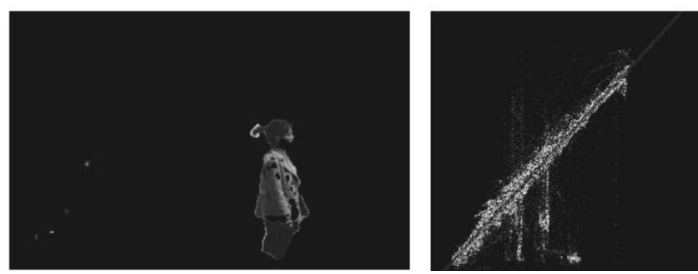

Fig. 12 Object detection using BMP in the case of drastic illumination change: (a) current image, (b) detection result and histogram using a single background mosaic, (c) detection result and histogram using BMP. The histograms in (b) and (c) are calculated between the corresponding background mosaic and the current image.

come the resolution change of images. There are lots of misdetections and false detections. On the other hand, the proposed method using each BMP as in Fig. 11(c) shows relatively good performance.

Figure 12 shows the effect of the BMP in the case of drastic illumination change on object detection. The sequence was obtained from a handheld CCD camera, and there is drastic illumination change, viz., a light turned on. Object detection is accomplished using the BMP shown in Fig. 9(a). We have three GIC levels, and the estimated illumination change is shown in Fig. 9(b). The result using a single background model as in Fig. 12(b) reveals that it cannot overcome the GIC compensation error. So there are lots of misdetections and false detections. From the histogram in Fig. 12(b), we can find that the GIC compensation error increased as the GIC got stronger if we used a single background mosaic. On the other hand, the proposed method using the BMP as in Fig. 12(c) shows relatively good performance.

\section{Video Coding Using the BMP}

A mosaic image obtained from all frames in an image sequence can be used in video coding ${ }^{26-32}$ as well as in object detection. The mosaic representation exploits large-scale spatial and temporal correlations in image sequences. So it performs substantially better than traditional interframe coding methods in many applications where there is significant camera motion. Another advantage of the methods using mosaics is random access. Mosaic-based video coding systems usually use two types of mosaics: static mosaics for storage, and dynamic mosaics for transmission. And some video coding systems utilize both types at the same time. In this work we are interested in a video codec using a static mosaic, and we can use the codec as the smallest unit in transmission. In other words, we do not have to transmit data every time when we encode each frame from an image sequence. We can transmit data for an image sequence at a time after encoding them, using the storage codec. In this work we are trying to utilize the results of an object detection algorithm for selective coding when there are drastic RC and GIC. In that case RC and GIC should be compensated also. But even if we use the codec using a mosaic and compensate RC and GIC, we cannot overcome some drastic changes with a single mosaic. So we propose a coding system using a BMP and use it for selective coding.

Figure 13 shows a block diagram for both encoder and decoder to be used at the same time in a video coding system using a BMP. The first thing to do is to generate a BMP with an estimated GM parameter and GIC parameter between each two adjacent background mosaics or between the corresponding background mosaic and each frame. Then the BMP and parameters are encoded and added to the bitstream. Finally, residuals from each frame and the reconstructed BMP with reconstructed parameters are encoded. The scheme is very similar to the conventional video encoder, ${ }^{33-38}$ except that it uses the background mosaic in the BMP as a reference image instead of the previous image and needs parameter coding. The decoding process is the reverse of encoding process, as shown in Fig. 13.

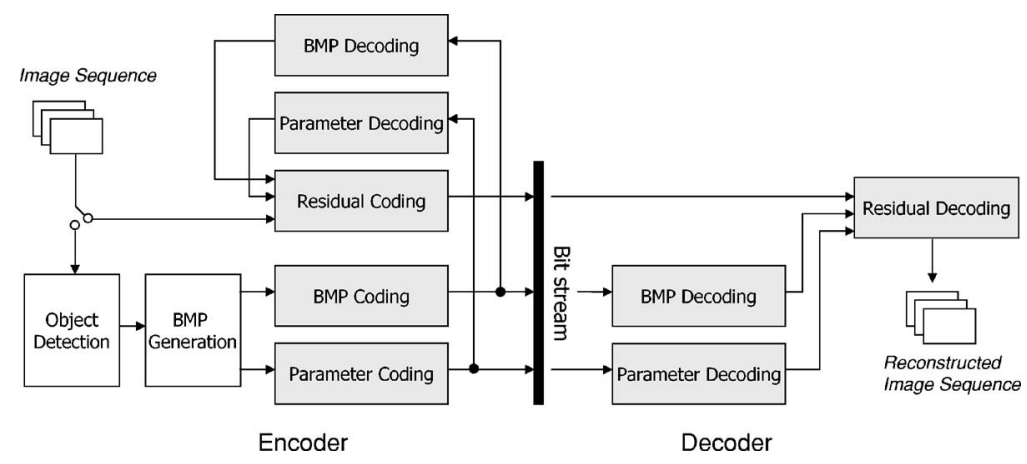

Fig. 13 Video codec using BMP. 


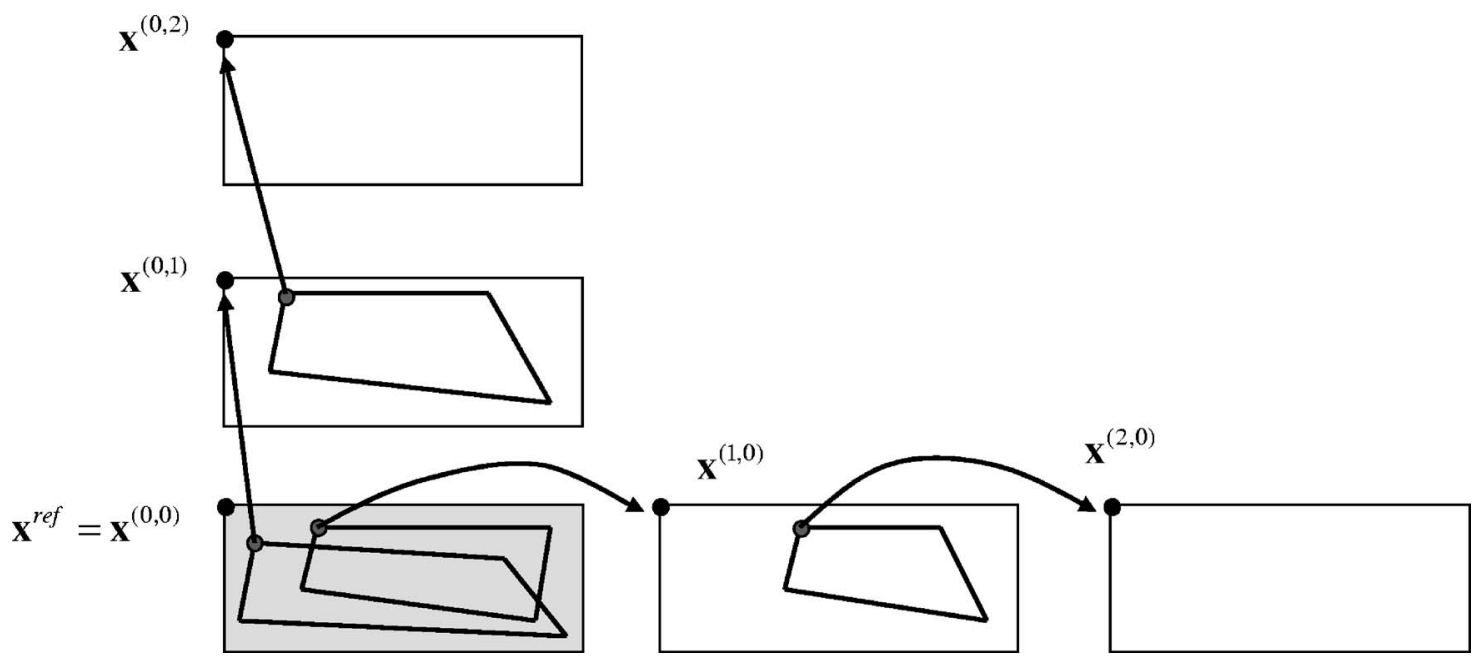

Fig. 14 BMP coding. The reference background mosaic is encoded using intra coding first, and the other background mosaics in the BMP are encoded using predictive inter coding. The arrows indicate prediction from each reference.

All the internal modules such as discrete cosine transformation, quantization, and entropy coding were implemented using H.263-based modules. ${ }^{34,37}$

\subsection{BMP Coding}

During the process of object detection in an image sequence, the BMP is generated dynamically as mentioned before. In order to encode residuals of images in the sequence, we should encode the BMP first. Figure 14 explains the process of BMP coding. The reference background mosaic is encoded using intra coding, and the other background mosaics are encoded using predictive inter coding. In Fig. 14 the arrows indicate the direction of prediction. For the background mosaic positioned at $(2,0)$ in the BMP, the residual is encoded between the prediction from the background mosaic at $(1,0)$ and the background mosaic at $(2,0)$. If there are two background mosaics that can be used as the reference of prediction, the one on the left has the first priority, e.g., for the background mosaic at $(2,1)$ the background mosaic at $(1,1)$ is used as the reference of prediction instead of the one at $(2,0)$. Of course, if there is only one background mosaic for the reference of prediction, that mosaic is used. All the predictions should be accomplished from the reconstructed background mosaics in the BMP, because the decoder should be able to reconstruct the same ones.

From some experimental results we find that the residual between each pair of background mosaics in the BMP is very important information, so that it must be treated carefully. In fact that is the main reason why we use the BMP instead of a single background mosaic.

\subsection{Parameter Coding}

GM and GIC parameters are used in this work, which are all real numbers, so they have to be quantized before storage or transmission. Without quantization, 32 bits are assigned to each parameter, and that is a waste of bits. So far, some researchers have chosen a method to allocate bits to each parameter on the basis of experimental results, ${ }^{39-42}$ but there has been no theoretical analysis for bit assignment. Now we need some strategies for the parameter coding. Even if the same quantization error occurs, it can cause different distortions in different applications. In other words, each application's induced distortion is more impor-

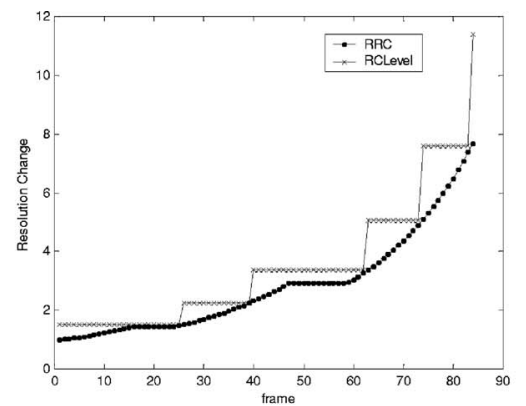

(a)

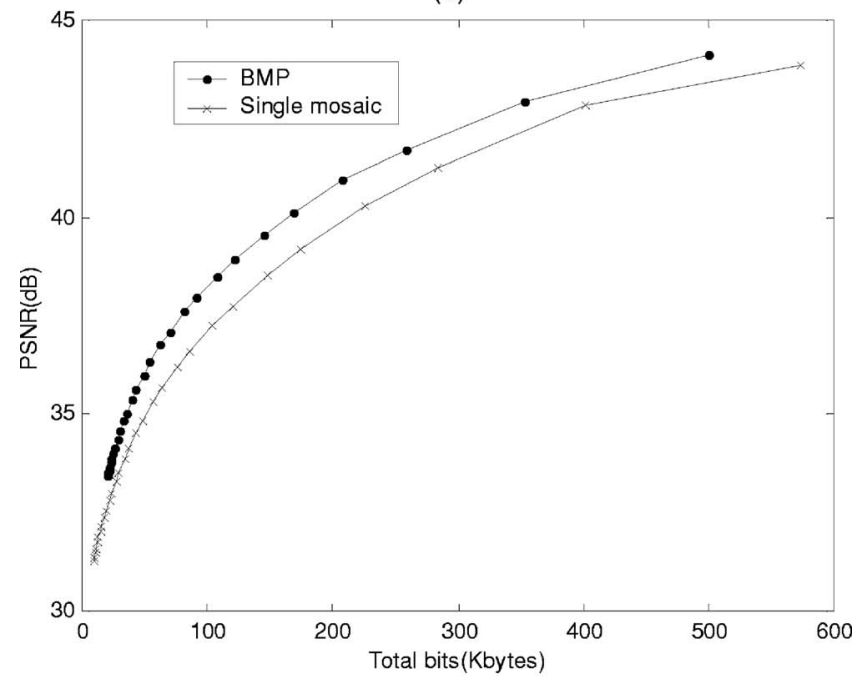

(b)

Fig. $15 \mathrm{RD}$ curve for an image sequence in a case of drastic resolution change: (a) resolution change, (b) RD curve using BMP. 


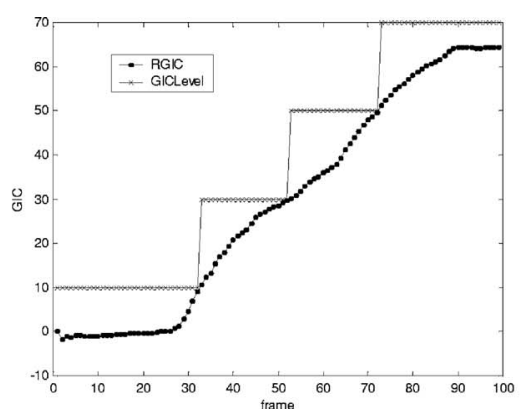

(a)

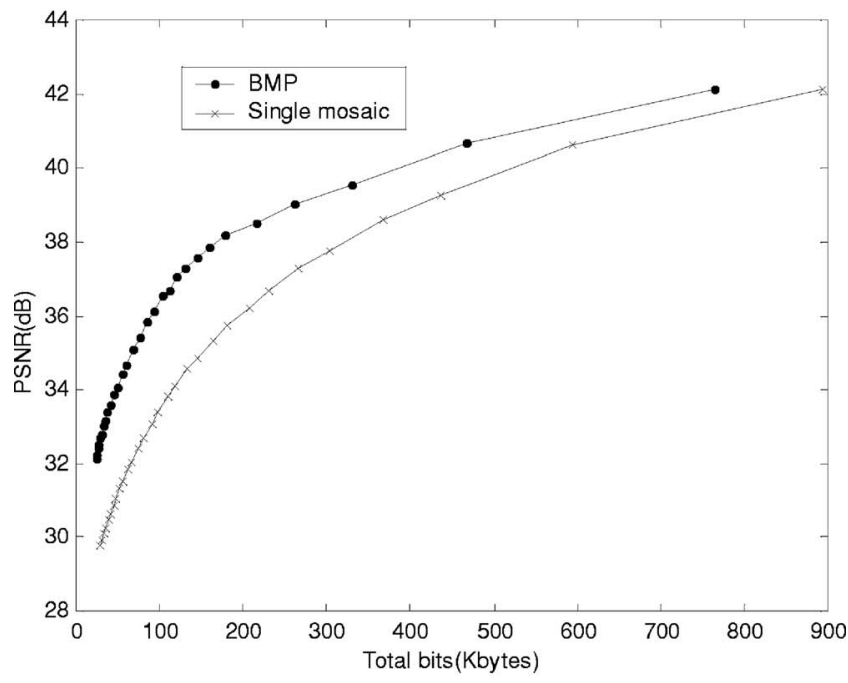

(b)

Fig. 16 RD curve for an image sequence in a case of drastic illumination change: (a) illumination change, (b) RD curve using BMP.

tant than the quantization error as such. So we must choose the quantization step size for each parameter after considering the induced distortion. In doing so, we need also some analytic methods instead of heuristic ones.

Another thing to be considered is each parameter's sensitivity. The most sensitive parameter can cause a drastic distortion when it has the same quantization step size as the other ones. In that case, we need to assign a much smaller quantization step size to that parameter than to the other parameters. The proposed parameter coding algorithm in Ref. 43 gives us each parameter's quantization step size when the maximum distortion of the application is given. So, if we know each parameter's dynamic range, we can encode each parameter. For the sequences used in this work, we assigned almost 60 bits to eight GM and two GIC parameters. Of course, a different number of bits were assigned to each parameter. For example, if the given total maximum distortion is 0.39 , five to seven bits were assigned to GM parameters, and four or five bits were assigned to GIC parameters.

\subsection{Experimental Results}

Figures 15 and 16 show RD curves in the case of drastic resolution change and illumination change, respectively. In both cases, we can see that the coding efficiency using the BMP is better than using a single background mosaic. In Fig. 16, almost twice as many bits are required to reach the

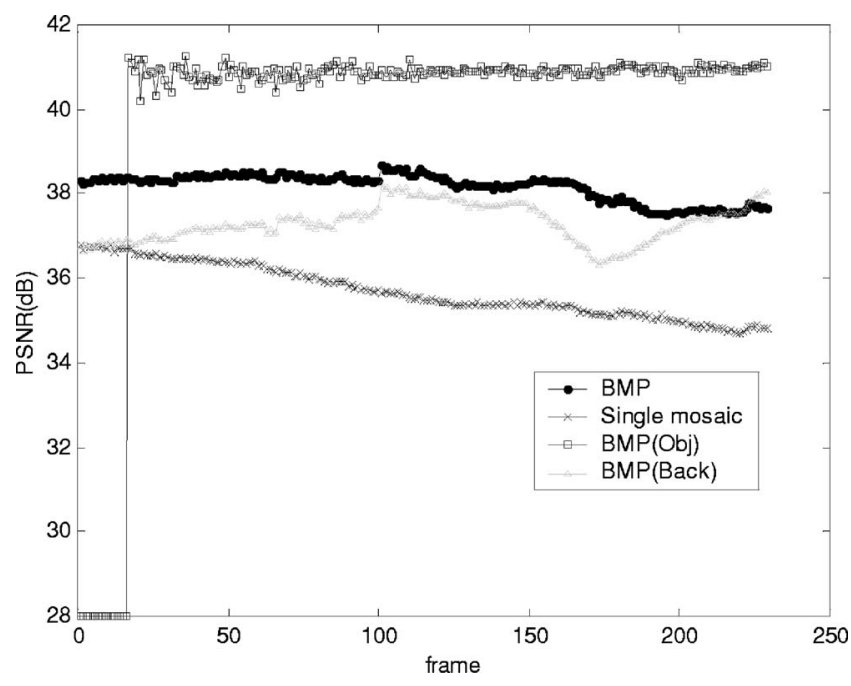

Fig. 17 PSNR of the reconstructed image sequence using selective coding with QP as in Table 2. BMP(Obj) means PSNR of the object blocks, and BMP(Back) that of the background blocks. A PSNR of $28 \mathrm{~dB}$ for the object blocks means that there is no object block in that frame.

PSNR of $38 \mathrm{~dB}$ if we use a single background mosaic. Of course, the total bits include the encoded bitstream for the mosaic itself as well as the residuals of images.

The results of object detection can be used not only when we generate the BMP itself but also when we design a selective coding system. The system assigns more bits to the object blocks than to the background ones, because the object region is generally more important. Here, an object block is a block that contains at least one object pixel in that block. Our strategy for the system is that we can increase the PSNR for the object region while keeping almost the same PSNR for the background region as when using a single background mosaic. Figure 17 shows the PSNR of the reconstructed images in the sequence with the QP specified in Table 2, which makes the same total number of bits. The PSNR for the object blocks is very high, and the PSNR for the background blocks also lies between those for the BMP and a single background mosaic. In other words, we took advantage of the BMP over a single background mosaic when designing a selective coding system for the case of drastic resolution and illumination change. A PSNR of $28 \mathrm{~dB}$ for the object blocks means that there is no object block in that frame. Figure 18 shows the reconstructed image using selective coding for 229th frame in the image

Table 2 QP used in selective coding for the sequence in Fig. 17.

\begin{tabular}{lc}
\hline \hline Block & QP \\
\hline BMP & 7 \\
Single mosaic & 9 \\
Object & 2 \\
Background & 11 \\
\hline \hline
\end{tabular}




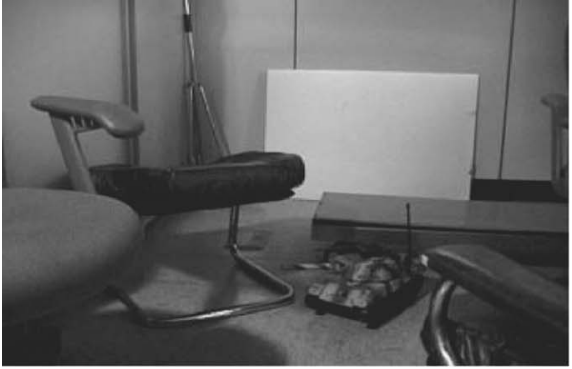

(a)

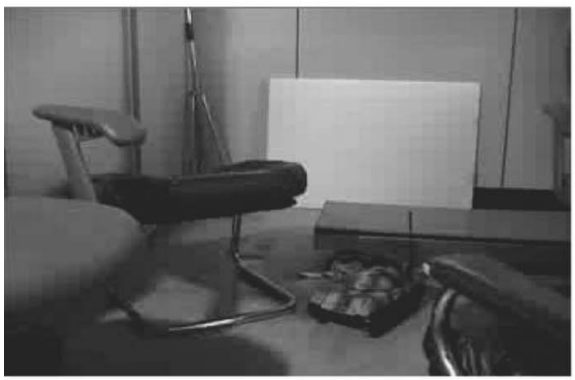

(c)

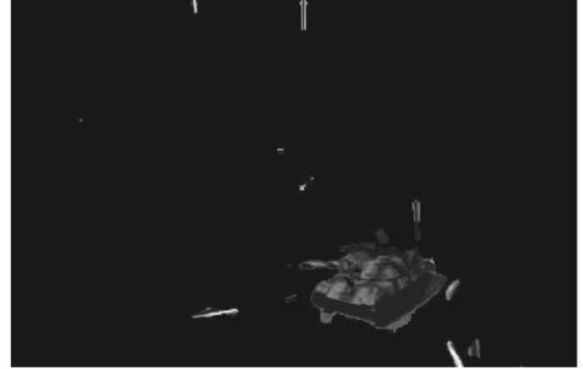

(b)

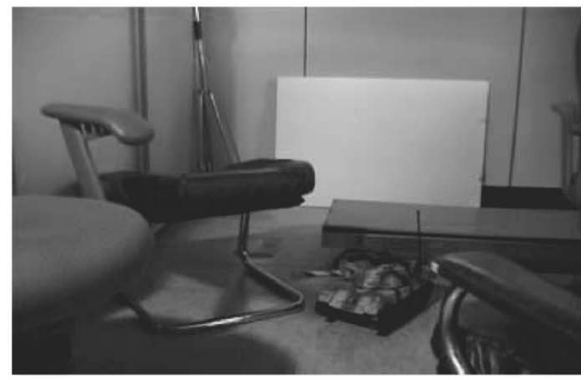

(d)

Fig. 18 The quality of reconstructed image of the sequence in Fig. 17: (a) 229th frame, (b) object mask, (c) reconstructed image using a single background mosaic, (d) reconstructed image using selective coding.

sequence in Fig. 17. Figure 18(d) shows better quality than Fig. 18(c) in the background blocks as well as in the object blocks.

\section{Conclusions}

In this paper we have proposed an algorithm for object detection in image sequences and developed a selective coding system using a background mosaic plane (BMP). Especially when the camera moves and zooms in on something to track a detected object, it is very useful to generate a background mosaic and use it for object detection instead of wasting the past parts of images. But, in generating a background mosaic and using it to detect objects, we observed two serious problems. One is resolution change, and the other is illumination change. These problems are very serious ones in video coding also. So, we proposed a multiple background mosaic system, called the BMP, and used it for object detection and video coding to overcome these problems. Some experimental results in various environments showed that the average performance of the proposed algorithm was good.

So far, we have been using the BMP for object detection and video coding originating from a single camera with lots of motions. But occasionally we also need to use a multiple-camera system. Each camera of the system communicates with the other cameras and can provide better performance for object detection and video coding using all the BMPs.

\section{References}

1. C. Stauffer and W. E. L Grimson, "Adaptive background mixture models for real time tracking," in IEEE Computer Society Conf. on Computer Vision and Pattern Recognition II, pp. 246-252 (1999).

2. S. Huwer and H. Niemann, "Adaptive change detection for real-time surveillance applications," in Third IEEE Int. Workshop on Visual Surveillance, pp. 37-46 (2000).
3. K. Toyama, J. Krumm, B. Brumitt, and B. Meyers, "Wallflower: principles and practice of background maintenance," in Int. Conf. on Computer Vision, Vol. 1, pp. 255-261 (1999).

4. M. Boninsegna and A. Bozzoli, "A tunable algorithm to update a reference image," Signal Process. Image Commun. 16, 353-365 (2000).

5. A. Elgammal, R. Duraiswami, D. Harwood, and L. S. Davis, "Background and foreground modeling using nonparametric kernel density estimation for visual surveillance," Proc. IEEE 90, 1151-1163 (Jul 2002).

6. E. P. Ong, B. J. Tye, W. S. Lin, and M. Etoh, "An efficient video object segmentation scheme," in IEEE Int. Conf. on Acoustics, Speech, and Signal Processing IV, pp. 3361-3364 (2002).

7. O. Javed, K. Shafique, and M. Shah, "A hierarchical approach to robust background subtraction using color and gradient information," in Proc. IEEE Workshop on Motion and Video Computing, pp. 22-27 (2002).

8. F. Ziliani and A. Cavallaro, "Image analysis for video surveillance based on spatial regularization of a statistical model-based change detection," in Proc. 10th Int. Conf. on Image Analysis and Processing, pp. 1108-1111 (1999).

9. L. Marcenaro, F. Oberti, and C. S. Regazzoni, "Multiple objects color-based tracking using multiple cameras in complex time-varying outdoor scenes," in 2nd IEEE Int. Workshop on Performance Evaluation of Tracking and Surveillance (2001).

10. P. L. Rosin, "Thresholding for change detection," in Sixth Int. Conf. on Computer Vision, pp. 274-279 (1998).

11. P. D. Picton, "Tracking and segmentation of moving object in a scene," in Proc. 3rd Int. Conf. on Image Processing Appl., pp. 389393 (1989).

12. J. S. Lee, K. Y. Rhee, and S. D. Kim, "Moving target tracking algorithm based on the confidence measure of motion vectors," in Proc. 2001 Int. Conf. on Image Processing, pp. 369-372 (2001).

13. J.-H. Cho and S.-D. Kim, "Object detection using multi-resolution mosaic in image sequences," Signal Process. Image Commun. 20(3), 233-253 (2005).

14. J.-H. Cho and S.-D. Kim, "Object detection using spatio-temporal thresholding in image sequences," Electron. Lett. 40(18), 1109-1110 (2004).

15. E. Trucco and A. Verri, Introductory Techniques for 3-D Computer Vision, Prentice-Hall (1998).

16. K. Skifstad and R. Jain, "Illumination independent change detection from real world image sequences," Comput. Vis. Graph. Image Process. 46(3), 387-399 (1989).

17. S. C. Liu, C. W. Fu, and S. Chang, "Statistical change detection with moments under time-varying illumination," IEEE Trans. Image Process. 7(9), 1258-1268 (1998). 
18. E. Durucan and T. Ebrahimi, "Change detection and background extraction by linear algebra," Proc. IEEE 89(10), 1368-1381 (2001).

19. S. Baker and I. Matthews, "Lucas-Kanade 20 years on: a unifying framework," Int. J. Comput. Vis. 56(3), 221-255 (2004).

20. H.-Y. Shum and R. Szeliski, "Panoramic image mosaics," Technical Report, pp. 1-50, Microsoft Research (1997).

21. J. Yao, "Image registration based on both feature and intensity matching," in Proc. 2001 IEEE Int. Conf. on Acoustics, Speech, and Signal Processing, Vol. 3, pp. 1693-1696 (2001).

22. M. Irani and P. Anandan, "Robust multi-sensor image alignment," in 6th Int. Conf. on Computer Vision, pp. 959-966 (1988)

23. H.-M. Chen and P. K. Varshney, "Automatic two-stage IR and MMW image registration algorithm for concealed weapons detection," IEE Proc. Vision Image Signal Process. 148(4), 209-216 (2001).

24. C.-W. Lee and S.-D. Kim, "Non-uniformly sampled mosaic construction using resolution map," Electron. Lett. 38(24), 1515-1516 (2002).

25. Y. Altunbasak, R. M. Mersereau, and A. J. Patti, "A fast parametric motion estimation algorithm with illumination and lens distortion correction," IEEE Trans. Image Process. 12(4), 395-408 (2003).

26. M. Irani, S. Hsu, and P. Anandan, "Mosaic-based video compression," in Digital Video Compression: Algorithms and Technologies 1995, A. A. Rodriquez, R. J. Safranet, and E. J. Delp, Eds., Proc. SPIE 2419, 242-253 (1995).

27. B. Tannenbaum, R. Suryadevara, and S. Hsu, "Evaluation of a mosaic based approach to video compression," in Conf. Proc., IEEE Int. Conf. on Acoustics, Speech, and Signal Processing, pp. 1213-1215 (1996).

28. M. Irani, P. Anandan, and S. Hsu, "Mosaic based representation of video sequences and their applications," in Proc. Fifth Int. Conf. on Computer Vision, pp. 605-611 (1995).

29. S. Hsu and P. Anandan, "Hierarchical representation for mosaicbased video compression," in Proc. PictureCodingSymp, pp. 1-6 (1996).

30. D. Farin, H. N. Peter, and W. Effelsberg, "Minimizing MPEG-4 sprite coding-cost using multi-sprites," Proc, SPIE 5308, 234-245 (2004).

31. S.-Y. Chien, C.-Y. Chen, W.-M. Chao, C.-W. Hsu, Y.-W. Huang, and L.-G. Chen, "A fast and high subjective quality sprite generation algorithm with frame skipping and multiple sprites techniques," in Proc. 2002 Int. Conf. on Image Processing, pp. 193-196 (2002).

32. W. H. Leung and T. Chen, "Compression with mosaic prediction for image-based rendering applications," in IEEE Int. Conf. on Multimedia and Expo III, pp. 1649-1652 (2000).

33. I. E. G. Richardson, H.264 and MPEG-4 Video Compression, John Wiley \& Sons (2003).

34. J.-K. Kim, Visual Communication System, Youngchi.

35. H. Hiroshi, The current MPEG, ASCII Corp.

36. ITU-T Recommendation H.261, "Video codec for audiovisual services at $64-1920 \mathrm{Kbits} / \mathrm{s} "$ (1993).

37. ITU-T Recommendation H.263, "Video coding for low bit rate communication" (1998).
38. ISO/IEC JTC1/SC29/WG11 N3312, "MPEG-4 Video Verification Model version 16.0" (2000)

39. J. Ostermann, "Object-based analysis-synthesis coding (OBASC) based on the source model of moving flexible 3-D objects," IEEE Trans. Image Process. 3(5), 705-711 (1994).

40. Y.-H. Ko, J. G. Choi, S.-J. Cho, and S. D. Kim, "Efficient coding algorithm for affine motion parameters," Opt. Eng. 40(2), 200-208 (2001).

41. M. Hotter, "Object-oriented analysis-synthesis coding based on moving two-dimensional objects," Signal Process. Image Commun. 2, 409-428 (1990).

42. K. Kamikura, H. Watanabe, H. Jozawa, H. Kotera, and S. Ichinose, "Global brightness-variation compensation for video coding," IEEE Trans. Circuits Syst. Video Technol. 8(8), 988-1000 (1998).

43. J.-H. Cho, "Object detection and video coding using background mosaic plane," Doctoral Thesis, Dept. of EE \& CS, Division of EE, KAIST (2006)

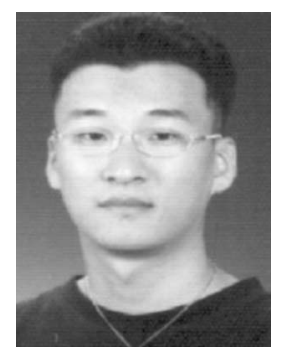

Ju-Hyun Cho received his BS, MS, and $\mathrm{PhD}$ degrees in 1999, 2002, and 2006, respectively, in the Department of EE \& CS, Division of EE, KAIST. His research interests include visual surveillance, video coding, and real-time object detection and tracking.

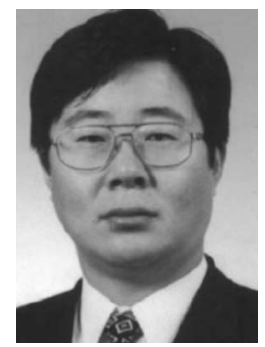

Seong-Dae Kim received the BS degree in electronic engineering from Seoul National University in 1977, and the MS degree in electrical engineering from KAIST in 1979. $\mathrm{He}$ received the Diplôma de DocteurIngénieur from Ecole Nationale Supérieure d'Electrotechnique d'Electronique D'Informatique et d'Hydraulique of the Institut National Polytechnique de Toulouse, France, in 1983. He joined the Department of Electrical Engineering at KAIST in September 1984 , and he is currently a professor there. His current research interests include pattern recognition, multidimensional signal processing, image coding, and very large scale integration (VLSI) realization of image-processing algorithms. 